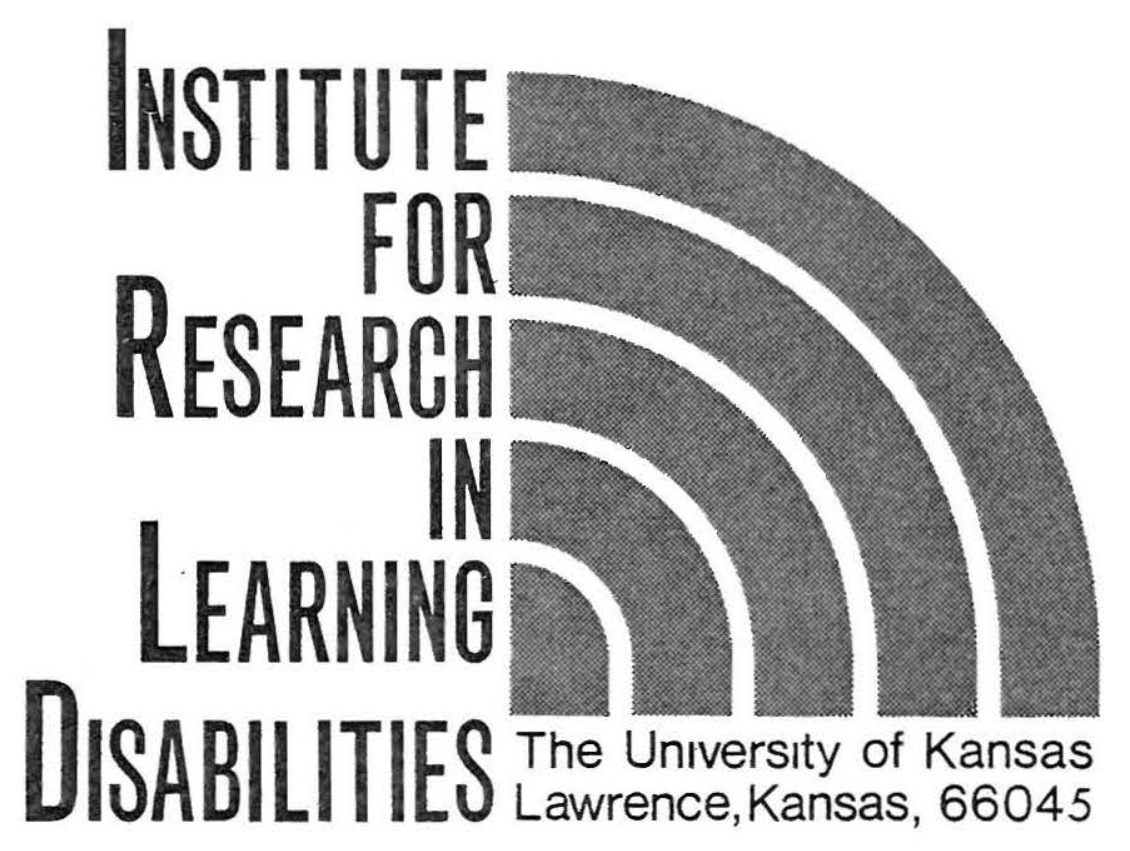

Emphasis on Adolescents and Young Adults

IDENTIFICATION OF LEARNING DISABLED ADOLESCENTS:

A BAYESIAN APPROACH

Gordon R. Alley, Donald D. Deshler, and Michael M. Warner

Research Report \#2

January, 1980

This study was conducted in cooperation with Lawrence USD 497, Lawrence Kansas. This report was originally published in the Learning Disability Quarterly, 1979, 2(2), 76-83. 
The University of Kansas Institute for Research in Learning Disabilities is supported by a contract (\#300-77-0494) with the Bureau of Education for the Handicapped, Department of Health, Education, and Welfare, U.S. Office of Education, through Title VI-G of Public Law 91-230. The University of Kansas Institute, a joint research effort involving the Department of Special Education and the Bureau of Child Research, has specified the learning disabled adolescent and young adult as the target population. The major responsibility of the Institute is to develop effective means of identifying learning disabled populations at the secondary level and to construct interventions that will have an effect upon school performance and life adjustment. Many areas of research have been designed to study the problems of LD adolescents and young adults in both school and non-school settings (e.q., employment, juvenile justice, military, etc.).

Co-Directors: Edward L. Meyen

Richard L. Schiefelbusch

Research Coordinator: Donald D. Deshler

Associate Coordinator: Jean B. Schumaker

Institute for Research in Learning Disabilities

The University of Kansas

313 Carruth-O'Leary Hall

Lawrence, KS 66045

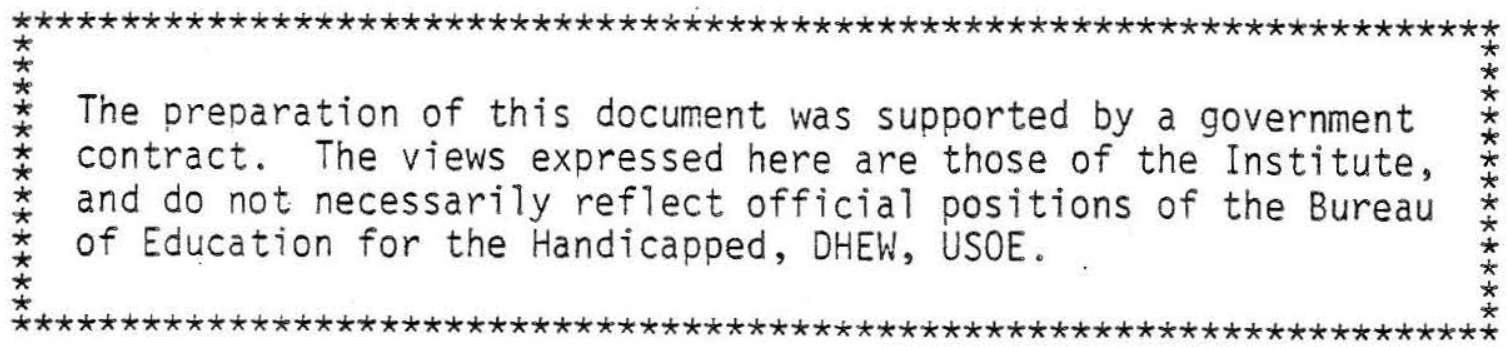




\section{Cooperating Agencies}

Were it not for the cooperation of many agencies in the public and private sector, the research efforts of The University of Kansas Institute for Research in Learning Disabilities could not be conducted. The Institute has maintained an on-going dialogue with participating school districts and agencies to give focus to the research questions and issues that we address as an Institute. We see this dialogue as a means of reducing the gap between research and practice. This communication also allows us to design procedures that: (a) protect the LD adolescent or young adult, (b) disrupt the on-going program as little as possible, and (c) provide appropriate research data.

The majority of our research to this time has been conducted in public school settings in both Kansas and Missouri. School districts in Kansas which are participating in various studies include: United School District (USD) 384, Blue Valley; USD 500, Kansas City; USD 469, Lansing; USD 497, Lawrence; USD 453, Leavenworth; USD 233, 01a the; USD 305, Salina; USD 450, Shawnee Heights; USD 512, Shawnee Mission, USD 464, Tonganoxie; USD 202, Turner; and USD 501, Topeka. Studies are also being conducted in Center School District and the New School for Human Education, Kansas City, Missouri; the School District of St. Joseph, St. Joseph, Missouri; Delta County, Colorado School District; Montrose County, Colorado School District; Elkhart Community Schools, Elkhart, Indiana; and Beaverton School District, Beaverton, Oregon. Many Child Service Demonstration Centers throughout the country have also contributed to our efforts.

Agencies currently participating in research in the juvenile justice system are the Overland Park, Kansas Youth Diversion Project and the Douglas, Johnson, and Leavenworth County, Kansas Juvenile Courts. Other agencies have participated in out-of-school studies-Achievement Place and Penn House of Lawrence, Kansas, Kansas State Industrial Reformatory, Hutchinson, Kansas; the U.S. Military; and the Job Corps. Numerous employers in the public and private sector have also aided us with studies in employment.

While the agencies mentioned above allowed us to contact individuals and supported our efforts, the cooperation of those individuals--LD adolescents and young adults; parents; professionals in education, the criminal justice system, the business community, and the military--have provided the valuable data for our research. This information will assist us in our research endeavors that have the potential of yielding greatest payoff for interventions with the LD adolescent and young adult. 


\title{
IDENTIFICATION OF LEARNING DISABLED ADOLESCENTS: A BAYESIAN APPROACH
}

\author{
Gordon R. Alley, Donald D. Deshler and Michael M. Warner
}

\begin{abstract}
The identification of learning disabled adolescents for program placement is a major concern of school personnel. An array of problems are associated with the identification of learning disabled populations ranging from the use of the best differentiating characteristics to the types of measures used. The identification model discussed in this article attempts to address some of these problems. The Bayesian approach is an alternative to traditional methods that rely primarily on psychometric data or classroom/clinical observation for identification decisions. D.D.D.
\end{abstract}

Certainly one of the critical needs facing professionals who work with learning disabled (LD) students is a reliable, efficient, and practical procedure for identifying these students in secondary school settings. The purpose of the present article is to present an identification approach which is designed to meet this need. It is based on a method called Bayesian Revision of Subjective Probabilities, named after the 18th century clergyman and mathematician, Thomas Bayes. Although widely used in fields such as engineering, medicine, business and economics, the potential for Bayesian methods is only beginning to be recognized in the field of education.

Initial investigations of the feasibility of using Bayesian procedures with elementaryaged LD students were reported by Wissink, Kass, and Ferrell (1975) and DeRuiter, Ferrell, and Kass (1975). While being based on those investigations, the identification approach discussed below extends them to ado- lescent populations and to the development of a screening instrument, the Component Disabilities Checklist (CDC). In addition, a screening process is described which combines data from the CDC with scores from formal tests to yield an estimate of the probability that a given student is at risk for LD.

It is believed that the approach to be presented has certain features which make it

GORDON R. ALLEY, Ph.D., is Professor, Department of Special Education, the University of Kansas.

DONALD D. DESHLER, Ph.D., is Associate Professor and Coordinator, the University of Kansas Institute for Research in Learning Disabilities.

MICHAEL M. WARNER, Ph.D., is Research Scientist, the University of Kansas Institute for Research in Learning Disabilities. 
very attractive to professional educators of LD adolescents. First, it does not rely on identification criteria which may be more appropriate to the identification of elementary-aged students; rather, it depends on judgments about, and data from LD and non-LD adolescent populations. Second, the procedures are efficient. Because decisions are based on data pertaining to a relatively small number of very diagnostic characteristics, large numbers of students can be screened in a relatively short time. Third, the procedures provide for a consistent and systematic approach to identification. If adopted by a school district, for example, the procedures result in a uniform set of screening criteria. As the LD teacher conferences with regular teachers and parents, justifications for the provision of LD services can be made consistently from one student to the next. Fourth, new information about students can be obtained and incorporated with prior information easily and as often as necessary, thus minimizing the possibility that a given student would remain misclassified for an extended period of time. Fifth, once they are made operational, the mechanics of the procedures are not complicated and require only the combined skills of the regular class teacher and someone qualified to administer individualized educational tests, such as the LD specialist.

In the simplest terms, the identification procedures are based on the following sequence of activities:

First, regular class teachers are asked to complete a checklist for each student experiencing difficulty in their classroom. This checklist consists of a limited number of weighted target behaviors that the classroom teacher is asked to observe in students. The weighted target behaviors included on the checklist have been estimated (by experts in learning disabilities) to be highly associated with the condition of learning disabilities in adolescent populations.

Second, since each factor on the checklist has been assigned a numerical weight (indicating that factor's odds of defining learning disability), a formula is used which combines odds data (or probability data). The formula, called Bayes' theorem, is used to determine the probability of a certain student being learning disabled based on the regular class teacher's responses on the checklist.

Third, if the resulting probability exceeds a certain cut-off point (indicating a high probability of the student being LD), the next step in the screening procedure is applied. This step consists of administering a limited number of psychometric subtests tapping the major target behavior areas in the checklist. This step in the screening procedure is designed to add precision to the judgments rendered by the regular classroom teacher. Student scores on each subtest are changed to probability measures so that using Bayes' theorem, this information can again be combined to yield a probability estimate of the student being learning disabled.

Finally, if student scores received on the subtests result in a high probability of $\mathrm{LD}$, the student is referred to the school staffing team for indepth diagnosis and confirmation of the screening decision yielded by the above steps. In short, this procedure is an attempt to increase the accuracy of screening decisions so that only those students with the highest probability of being learning disabled receive the full battery of achievement, diagnostic, and aptitude tests for LD placement and programming.

The following discussion is organized into three sections: (a) a rationale for the identification procedures being advocated, (b) an extended description of the procedures and their development, and finally, (c) suggestions for future directions of the development of the procedures.

\section{RATIONALE}

It is assumed that some characteristics provide more information than others regarding the differentiation of LD and non-LD students and that, to be efficient, an identification procedure should collect data only on a small number of these most discriminating characteristics. A productive strategy would seem to be one which culled from the many reported characteristics of LD adolescents those that were at the same time most typical of LD students and least typical of non-LD students.

Another assumption is that an appropriate source of knowledge of the most differen- 
tiating characteristics is the judgments of trained LD specialists, particularly those who have experience teaching both special and regular classes. The Bayesian Revision of Subjective Probabilities is a quantitative application of a cognitive process. Using Bayesian statistics, it is assumed that "people observe other individuals and events and, in the process ... acquire knowledge and information" (Wittrock, 1977, p. IX).

The task is to delineate the salient variables that the LD expert uses when identifying LD adolescents. These variables must then be ranked according to their power to differentiate an LD adolescent. The higher the odds that the presence of a given factor signals $L D$ rather than non- $L D$, the more differentiating is the factor.

It is also assumed that "in their heads" LD experts have knowledge of component disabilities and their respective probabilities. Therefore, a cognitive search of the LD experts is required for this information before others, i.e., nonexperts in LD, can use the information to screen for LD. Using this information, a nonexpert (e.g., the regular classroom teacher) may approximate the odds ratios of the LD expert when screening for LD among adolescents.

A closely related assumption is that the differentiating characteristics, described in terms of behaviors familiar to the regular class teacher, could be accurately judged by that teacher as being present or absent in a given student. It is believed that, due to their daily contact with students, regular class teachers are in an excellent position to make such judgments.

Consistent with the Bayesian orientation, it is further assumed that data additional to current test scores or checklist responses should be considered in the decision-making process. For example, an important piece of prior information is the percentage of the total student body that a district allows to be called LD for the purposes of providing services. Thus, if a $2 \%$ figure was established, a different group of students would be identified as being LD than if an $8 \%$ figure was established. It is important to include such information in identification decisions since it helps to define the parameters of the situation.
In addition, as new information is acquired about a student, such new information should not be considered in isolation. Rather, it must be combined with older data to derive revised estimates of the probability that a student is LD. Bayes' theorem provides a convenient mathematical mechanism for combining such data in a formal and consistent way.

Next, it is assumed that it is appropriate and meaningful to describe the condition of LD in terms of a probability statement. Such a statement can be interpreted as our "degree of belief" or our "best bet" that a given student is LD. The practitioner may state, for example, "Knowing that this adolescent has been retained in the second grade, has word recognition problems, and is currently enrolled in a remedial writing course in junior-high school, the odds are 4:1 that the adolescent is LD". Of course, the probability that the adolescent is LD can vary based on (a) knowledge of the weight (importance) that should be assigned to the given information, and (b) increasing the amount of information about a given student that can be used to change one's probability estimate of the youngster being LD. The probabilistic method described here can account for both presence or absence of data and can be used to weigh and combine the data that are present.

Readers who have been steeped in the tradition of the "objectivity" of formal test data may find discomforting the "game of chance" orientation presented here. Nevertheless, uncertainty and subjective judgment are associated with any human decision and as Novick and Jackson (1974) have pointed out, there is always a possibility of error on any measure of human behavior.

Another assumption central to the identification process described in this paper is that the students who are the most appropriate candidates for LD services are those who exhibit an aggregation of performance deficits. The student whose only noticeable difficulty is in the area of decoding words or spelling, for example, will not be identified as LD by the procedures described herein. However, this does not appear to be a serious limitation of the procedures. First, it is rare that the student with only one specific disability is so dysfunc- 
tional in the school setting that intensive and/or extensive special education intervention is required. Rather, as disabilities become multiple they increasingly limit the students' options for compensation using their personal resources. Consultative assistance may be more appropriate for the student with an isolated problem. Second, if a single disability is so severe as to significantly interfere with the student's school performance, that student is likely to come to the attention of appropriate school personnel through coexisting screening procedures such as teacher referral and/or low grade reports.

A final assumption is that each disability that is formally considered is statistically independent from every other disability that is considered. For the present purposes this means that knowing a student was identified with one component disability (e.g.; a disability in decoding words) would not influence our estimate of whether or not that student would be identified with another component disability (e.g., a disability in detecting spelling errors). This assumption allows one to use simple and manageable mathematical formulas to combine information about component disabilities. Yet, strictly speaking, the condition of statistical independence could not be met in most of the practical situations of education where dependence of variables is the rule rather than the exception. DeRuiter et al. (1975) provided evidence that violation of this assumption may not result in serious consequences, especially if the component disabilities are relatively independent.

\section{DETERMINATION OF DIFFERENTIATING CHARACTERISTICS}

This section outlines three major steps that were followed to specify those characteristics which best differentiate LD adolescents from non-LD adolescents.

\section{Step I - Specification of Characteristics}

Associated with LD Adolescents

In order to determine which characteristics are most effective in identifying an LD adolescent population, one must first specify the range of characteristics which have been found to be associated with LD adolescents. To do this, the present authors conducted a thorough search of the literature for disabilities found to be related to LD in adolescents by empirical research and/or intuitive judgment based on observations in clinical settings. This search resulted in a list of more than 90 characteristics. Noted overlap was eliminated through combination, thereby reducing these factors to sixty-seven somewhat independent characteristics or component disabilities (CD).

These sixty-seven component disabilities (which represented all factors which the literature specified as having some relationship to LD in adolescent populations) were organized into a Component Disability Ques. tionnaire used to determine which of the factors were most effective in differentiating LD from non-LD. In order to obtain this information, a group of thirty-two LD specialists ${ }^{1}$ were asked, on the basis of their experience and knowledge of adolescents, to rate the prevalence of each component disability among LD and non-LD adolescent populations. Table 1 presents some sample component disabilities from this questionnaire. The medians for each component disability were calculated for the prevalence estimates provided by the group of specialists. The median prevalence estimates were used to calculate the likelihood ratios (LR) for each component disability. A likelihood ratio (LR) may be thought of as a weight assigned to a given component disability. Thus, a high LR indicates that a given component disability contributes more to identifying LD than a lower LR. The LR for each component disability is determined by simply dividing the median percentage estimate of the component disability in the LD population by the median percentage estimate of the component disability in the non-LD (NLD) population. Thus, in Table 1 the LR for a disability in decoding words is determined by dividing $80 \%$ by $20 \%$ which yields an LR of 4.0. An example of a characteristic that does not differentiate as effectively as a disability in decoding words is the second example provided in Table 1 , a disability in awareness of place. The LR of this component is 2.0; thus it is only twice as likely to be present in LD as in NLD populations.

The LRs for each of the sixty-seven components in the questionnaire were determined 


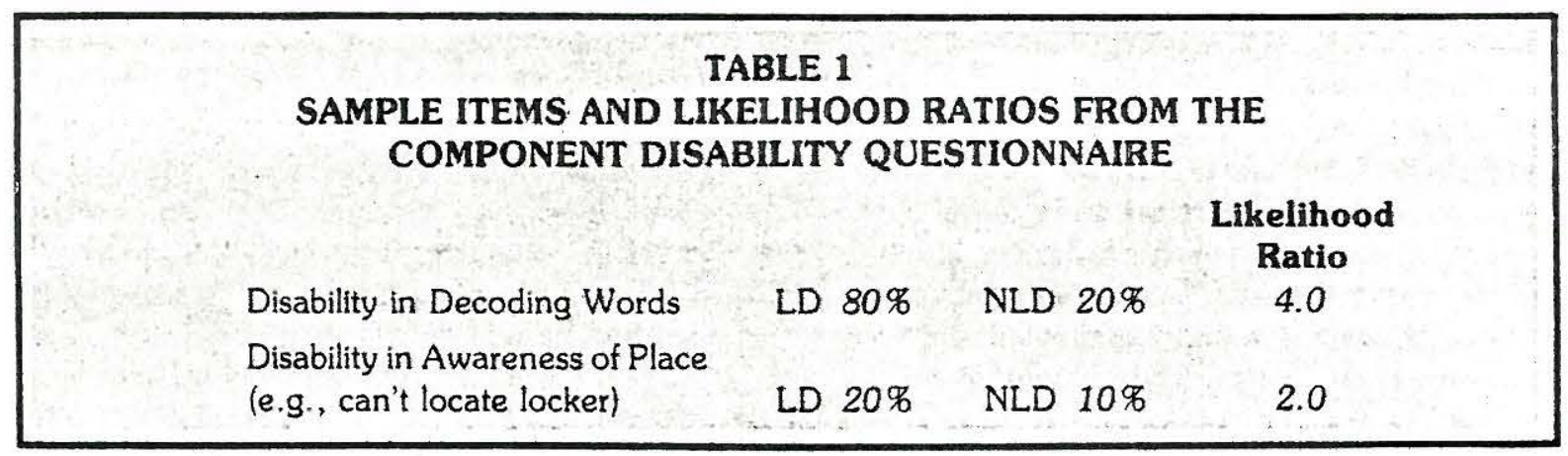

and ranked from highest to lowest. Table 2 demonstrates how the Bayes' formula uses the LRs from the four top-ranked component disabilities to yield a probability statement as to the degree of certainty that LD has been identified. Since the main function of Bayes' formula is to combine weighted information to give an aggregate estimate of the presence or absence of LD, each of the highest ranked component disabilities was put through the Bayesian formula. With the addition of each component disability, the estimate of LD is revised. In this case, if a youngster is found to have a deficit in the top four CDs, Bayes' formula combines the weighted $C D$ s to yield an LD probability of 96 . Bayes' formula will obviously work in the same fashion for any kind of weighted data given to it. However, if $\mathrm{CDs}$ with lower LRs are used (that is, characteristics that are not effective in differentiating LD from NLD) it will take more CDs to reach an equal level of certainty (96\%) that LD had identified. The four component disabilities that LD specialists found to best differentiate LD from NLD at the secondary level are: (1) disability in decoding words, (2) disability in recognizing sight words, (3) disability in detecting spelling errors, (4) disability in the use of mathematical algorithms (i.e., disability in being able to set up a problem for its solution).

\section{Step II - Putting the Differentiating}

Components into a Usable Form for Referral

Regular classroom teachers hold the major responsibility for identification and referral of the LD adolescent. Therefore, the next step in the development of the identification procedure was to design an instrument to be used by regular class teachers in making referrals from their classroom. An efficient screening procedure is one that gathers data only on target behaviors - that is, on those behaviors that have the highest probability of identifying a given population. Thus, the top four component disabilities discussed in Step I were used in the development of the screening instrument, Component Disability Checklist (CDC).

The $C D C$ was made up of a list of observable behaviors that the regular classroom teacher could see manifested by students performing classroom tasks. Each of the observable behaviors was related to one of the component disabilities. For example, for the component disability, "Disability in decoding words", the four observable behaviors included on the checklist were: (1) Unable to sound out words, (2) Unable to use structural clues (e.g., prefixes) to unlock words, (3) Unable to use context clues as aids in unlocking words, (4) Unable to read fluently. For each behavior on the checklist the teacher was asked to merely check "yes" or "no" to indicate whether or not a student evidenced that behavior.

The $C D C$ consisted of a total of 29 items. The purpose of the $C D C$ was to give to regular classroom teachers an instrument that allowed them to focus on salient target behaviors for a decision. The information included in the checklist was neither redundant nor tangential. It was not redundant because it included only those factors that were necessary to identify $L D$ with a certain degree of certainty (i.e., .96). Once that level was reached, additional data were not added. Thus, only four components were used. The information was not tangential because it did not include items assumed to be indirectly associated with LD in adolescents but included only those factors that had earlier been demonstrated to be highly related to LD.

The $C D C$ was given to all ninth-grade 


\title{
TABLE 2 \\ APPLICATION OF BAYES' FORMULA USING COMPONENT DISABILITIES THAT BEST DIFFERENTIATE LD FROM NLD AT THE SECONDARY LEVEL
}

\author{
Given: Bayes' Formula $\mathrm{p}(\mathrm{LD})=\underline{\mathrm{p}}\left(\mathrm{LR}_{1} \times \mathrm{LR}_{2} \times \ldots \times \mathrm{LR}_{\mathrm{n}}\right)$ combines \\ pp $\left(\mathrm{LR}_{1} \times \mathrm{LR}_{2} \times \ldots \times \mathrm{LR}_{\mathrm{n}}\right)+1$
}

prior and current information to determine the probability of $L D$.

Prior Information (pp). When making a decision on whether a youngster is/is not LD, we know some conditions/parameters upon which a decision is made. Specifically, we know how much of the total school population can be called (LD), e.g., a district policy may be that only $2 \%$ can be called LD. Therefore, the prior probabilities (pp) are.02.

Decisions concerning LD should not be made independent of this information.

Current Information $\quad\left(\mathbf{L R}_{\mathbf{1}} \ldots . \mathbf{L} \mathbf{R}_{\mathbf{2}}\right)$. This information is provided by observations made by the regular classroom teacher of the student's performance. In the case of the four top CDs, the LRs would be decoding words (6.0), recognizing sight words (4.4), detecting spelling errors (4.0), and mathematical algorithms (4.0).

\section{Aggregation of Component Disabilities with Bayes' Formula}

\section{Component Disability}

Decoding Words

Decoding Words + Reading Recognition

Decoding Words + Reading Recognition +

Detecting Spelling Errors

Decoding Words + Reading Recognition +

Detecting Spelling Errors + Math

Algorithms
Posterior Probability or p(LD) language arts teachers in USD $\# 497$ (Lawrence, Kansas) with instructions to complete it for every student in their classes. ${ }^{2}$ Four hundred fifty-four ninth-grade students were screened by the language arts teachers in one-half day. Thirty-five youngsters received checks in behaviors related to three or four of the component disabilities. This number represented $7.7 \%$ of the ninth-grade class.

The application of the checklist provides a preliminary indication of which adolescents within a school setting are at significant risk of being LD. However, the CDC does not pro- duce precise measures of the deficits a youngster is experiencing. Therefore, to increase the precision of the identification process, Step III becomes necessary.

Step III - Increasing Precision in the Identification Procedure

In order to increase precision over that provided by the $C D C$, a set of psychometric tests were selected to tap each of the component disability areas. The degree of precision added by this step can be readily seen by considering the component disability of "decoding words". On the checklist this component 
disability was represented by four observable behaviors which classroom teachers were to react to. To tap this component disability, two subtests were used: Word Attack Subtest of the Woodcock Reading MasteryTests and the Reading Efficiency Subtest of the lowa Silent Reading Test (lowa). The first subtest was used to measure the student's ability to apply specific rules in decoding words, whereas the lowa subtest was used to measure the student's ability to use context clues to decode words. Combined, these two subtests provide 33 probes of behavior. Thus, the precision of measurement has been increased by using such tests or subtests which tap the specific component disabilities. Table 3 lists the subtests used for each of the component disabilities.

Scores obtained on the psychometric subtests are translated into weighted LRs which can also be aggregated in Bayes' formula. Figure I describes the steps involved in this procedure as well as the way in which LRs were determined for all test scores for the four component disabilities.

To increase the precision of the identification decisions made by the ninth-grade language arts teachers in USD \#497, the thirtyfive students identified by these teachers through the $C D C$ were administered the subtests listed in Table 3 . The posterior probabilities derived from the administration of these psychometric tests resulted in nine youngsters (i.e., $2.35 \%$ of the ninth-grade students considered) being identified as LD.

\section{FUTURE DEVELOPMENTS}

The process of Bayesian identification has been described as being at the initial stages of development. In the future the following questions need to be investigated:

1. How reliable are the procedures associated with the different steps in the Bayesian identification process?

2. How much agreement can or should be obtained among groups of experts and what are the implications of nonagreement?

3. Taking the lead from DeRuiter et al. (1975), how do the present Bayesian procedures compare to other methods which do not assume that component disabilities are statistically independent, such as multiple regression and discriminate analysis?

4. How well do the present procedures discriminate LD adolescents from other groups with learning problems such as mentally retarded and behaviorally disordered students? Can the procedures be expanded to make these discriminations?

5. In what ways do students identified by the present screening procedures differ from those students identified using traditional procedures such as teacher referral?

6 . How can the present procedures be incorporated into a more general decision-theory framework? In other words, the possibility should be explored of formally describing the objective and subjective benefits or losses associated with correct and incorrect classifi-

\section{TABLE 3 \\ PSYCHOMETRIC SUBTESTS FOR THE TOP FOUR COMPONENT DISABILITIES}

\author{
Component Disability \\ Disability in Decoding Words \\ Disability in Recognizing \\ Sight Words \\ Disability in Detecting \\ Spelling Words \\ Disability in Mathematical \\ Algorithms
}

\section{Subtest}

Woodcock Reading Mastery Tests .

Word Attack Subtest

lowa Silent Reading Test .

Reading Efficiency Subtest

Peabody Individual Achievement

Test - Word Recognition Subtest

Stanford Achievement Test Spelling

Ross Test of Higher Cognitive

Processes - Test of Relevant

and Irrelevant Information 
Selection of Test Instruments: In this study an attempt was made to select tests which, in combination, would confirm the presence or absence of the condition of LD. Tests were selected which seemed to operationally define the four component disabilities which best discriminate LD from NLD students. Considetation was given to rellability of instruments and administration time.

Selection of Experimental Population: In order to determine likellhood ratlos for each possible test score for the selected subtests. it was necessary to administer the selected tests to LD and NLD populations. LD and NLD populations were chosen at the junior-high school level (Grades 7.9; $n=30 L D_{1}=30 \mathrm{NLD}$ ) and the senior-high school level (grades 10-12;n=30LD, $=30 \mathrm{NLD}$ ). To be included in the LD sample a student had to meet four ctiteria: (1) He/she was defined and placed by the school district for LD services; (2) He/she evidenced a disability in each of four component disability areas as judged by the student's LD teacher (teachers applied the criterion of a disability existing in each area if they feit it was stgnificant enough to create a major interference in learning): (3) He/she had an 1Q within the normal range (85-115); (4) $\mathrm{He}$ /she was not mentally retarded, emottonally disturbed or culturally different as judged by the LD teacher. Each of the LD students meeting the above criteria and NLD students not meeting the criteria were administered the selected subrests. Test scores from these administrations were used for the next four steps.

Establishing Prior Probabitities: Prtor probabillties represent the stale of knowledge prior to observations of a specific event of condition. They refes to the probability that any one of a number of possible mulually exclusive and exhaustive hypotheses is true. For this study, two hypotheses were used: (1) the hypothesis that learning disability is present, $P_{1 D}$. and (2) the hypothesis that nonieorning disobillty is present,

$\mathrm{P}_{\mathrm{NLD}}$. In this study exactly one-half of the students in the total sample were learning disabled and one-half were nonlearning disabled. Therefore, the prior probability used for the calculation of posterior probabilities in this study was .50 for each group. The prior probability varies according to the estimate of the total population being LD (e.g. If a district estimates that $3 \%$ of its population is LD the priors are $P_{L D}=.03$ and $\mathrm{P}_{\mathrm{NLO}}=.97$ )

Determination of Beta Distributtons: When a variable ts limited in range, the Beta distribution provides a useful model for lts distribution. In this study, the test scores were limited in range because of the limited sample size. Beta distributions were obtained for each sample group using a computer program designed for this purpose. The likethood that any score indicates the presence of learning disability is ascertained (1) by determining the height of the Beta curve at a particular score on a test, and (2) by determining the ratio between the height of the learning disability curve and the nonlearning disability curve at that score. The primary use of the Beta curves is to calculate likelihood ratios, not to interpret the curves themselves.

Calculation of Likelihood Ratios: As noted before, Beta distributions are derived for the purpose of calculating likelihood ratios (LR). LRs were derived for each student's score by dividing the height of the ordinate at that score in the LD Beta distribution by the height of the or. dinate at that score in the NLD distribution. The formula is: $L R(X)=p(X)_{L D}$

$$
\overline{p(X)} \text { NLD }
$$

$A L R$ of exactly one means that the ratio between the probability of that score in the LD sample and the probabillty of that score in the NLD sample is cne to one. LR of more than one indicates LD. LR of less than one indicates NLD.

Calculation of Postertor Probabilities: In this study posterior probabilities represent probability estimates that learning disability is pre. sent given a particular set of test scores. Posterior probabilities are cakculated by using the following formula:

$p(L D)=p D\left(L R_{t} \times L R_{z} \times \ldots \times L R n\right)$

$$
p p\left(L R_{1} \times L R, \times \ldots \times L R n\right)+1
$$

Where: $p(L D)=$ probabilittes of $L D$

$P P=$ prior probability estimate

$L R=$ likelihood ratio for each test score

cation (Hayes, 1973; Novick \& Jackson, 1974).

Because these questions about Bayesian identification remain unanswered at the present time, it is recommended that the approach be used only on a trial basis. Along with future refinements, the approach may provide school personnel with a practical and efficient method for identifying those adolescents who are in need of LD services.

\section{REFERENCES}

DeRuiter, J.A., Ferrell, W.R., \& Kass, C.E. Learning disabilities classification by Bayesian aggregation of test results. Journal of Learning Disabilities, 1975, 8, 365-372.

Hayes, W.L. Statistics for the social sciences (2nd ed.). New York: Holt, Rinehart, \& Winston, 1973.

Novick, M.R., \& Jackson, P.H. Statistical methods for educational and psychological research. New
York: McGraw Hill, 1974.

Wissink, J.F., Kass, C.E., \& Ferrell, W.R. A Bayesian approach to the identification of children with learning disabilities. Journal of Learning Disabilities, 1975, 8, 58-66.

Wittrock, M.C. (Ed.). Learning and instruction. Berkeley, Calif.: McCutchan, 1977.

\section{FOOTNOTES}

: The thirty-two specialists were LD teachers in junior and senior high schools in urban, suburban, and rural districts. The majority of these teachers held both full certification in learning disabilities and a master's degree in learning disabilities. All had experience teaching nonhandicapped adolescents as well as LD adolescents.

${ }^{2}$ The authors wish to acknowledge the cooperation of Dr. Donald W. Herbel, Director of Special Education, and the principals and teachers in the junior and senior high schools of USD $\# 497$. Lawrence, Kansas. 